\title{
Charge Violation and Alice Behavior in Global and Textured Strings
}

\author{
Katherine M. Benson \\ Department of Physics \\ Emory University \\ 1510 Clifton Road, NE \\ Atlanta, GA 30322-2430
}

(November 18, 2018)

\begin{abstract}
Spontaneous breaking of global symmetries can produce "Alice" strings: line defects which make unbroken symmetries multivalued, induce apparent charge violation via Aharonov-Bohm interactions, and form point defects when twisted into loops. We demonstrate this behavior for both divergent and textured global Alice strings. Both adiabatically scatter charged particles via effective Wilson lines. For textured Alice strings, such Wilson lines occur at all radii, and are multivalued only inside the string. This produces measurable effects, including path-dependent charge violation.
\end{abstract}




\section{INTRODUCTION}

Among the defects created in symmetry-breaking phase transitions are Alice strings. [1] 20 Like monopoles [4], Alice strings obstruct the global extension of unbroken symmetries, making them multivalued when parallel transported around the string. This algebraic obstruction has two prominent physical consequences. First, it produces nonconservation of the associated charges under Aharonov-Bohm scattering around the string. Second, it induces topologically stable point defects, which are themselves twisted loops of Alice string.

These Alice features were first described for gauged strings, where a charged condensate winds asymptotically due to gauge flux on the string. The gauge flux acts asymptotically on all charged particles in the same way, through the Wilson line $U(\varphi)$. Thus the condensate's winding, which makes Alice symmetries multivalued, is communicated to all charged particles circumnavigating the string. This communication induces both results above: Aharonov-Bohm scattering around the string changes both charge and monopole number. Loops of string, which leave charge and monopole number well-defined asymptotically, thus support deposited charge ("Cheshire charge") and deposited monopole number. [1.2] We have recently established, through topological arguments, that Alice loops can always carry such deposited monopole number by twisting. [5]

More recent work confirms that Alice phenomena also occur for global strings. [6 \& Such strings arise when global symmetries - describing flavor, chiral, or condensed matter degeneracies - break down. Two types of global strings can form: divergent strings, which wind nontrivially far from the string and thus have logarithmically divergent tension; and textured strings, which approach constant field values asymptotically, but carry topological winding in their finite tension cores.

We note here that divergent global strings inherit their Alice characteristics from the gauged and textured cases. Their topological structure coincides with the gauged case: therefore the same topological arguments identify twisted Alice loops with global monopoles.

[5] Their couplings to fundamental charged particles are the same as those of their textured 
cousins. Our careful Aharonov-Bohm analysis of these interactions shows that divergent strings induce an effective Wilson line for point charges, usually equivalent to the Wilson line for the gauged case. Thus divergent global Alice strings possess the entire constellation of Alice features: charge-altering Aharonov-Bohm scattering (first noted in [8], [7]), as well as twisted loops which are point defects.

Recently Benson, Manohar and Saadi found textured global strings with the same features. [6] At finite radius, their strings alter charges of particles that Aharonov-Bohm scatter around them. They also form twisted loops which are skyrmions, just as gauged Alice strings form twisted loops which are monopoles.

We show here that these Alice properties emerge generically for textured Alice strings. The result is striking, as it stems from very different physics than that of the the gauged case. The topology differs: gauged strings have nontrivial first homotopy group $\pi_{1}$, and form twisted loops with nontrivial $\pi_{2}$; while textured strings have nontrivial $\pi_{2}$, and form twisted loops with nontrivial $\pi_{3}$. Gauged strings vary nontrivially at infinite radius, yielding long-range flux-mediated interactions; while textured strings are asymptotically constant, yielding short-range direct interactions. Yet both are line defects with the Alice constellation of features: they induce apparent charge violation in Aharonov-Bohm scattering of charged particles (at least at finite scattering distances), and they form twisted loops that are topological point defects.

We demonstrate these results below as follows. The divergent global Alice string has the simplest structure, and thus appears in section $\mathbb{1}$. Here we construct the divergent Alice string, assuming that the string couples naturally to fundamental charges via effective mass terms. We then show how global Aharonov-Bohm scattering converts such couplings into an effective charge-violating Wilson line related to that of the gauged case. This analysis generalizes earlier work of McGraw [7], and lets us develop tools for studying textured string scattering in a simpler context.

We then consider the textured Alice string. We construct it topologically in section ПII, again assuming representations with effective mass couplings to the fundamental charges. 
Section IV explores the scattering of point charges in the textured string background. This scattering — which generically mixes degenerate states through a nonabelian AharonovBohm effect - has a rich structure. It induces an effective Wilson line $U(r, \varphi)$ which is radially dependent: while trivial at infinite radius, it becomes a multivalued group rotation at finite radii. This induces multiple effects. At finite radius, eigenstates of locally unbroken charge alter their charge, in an $r$-dependent way, in circumnavigating the string. Even asymptotic unbroken charges can interact non-trivially with the string, by approaching it, circumnavigating it at finite radius $r_{o}$, and then returning with altered charge.

Finally, in section $\mathrm{V}$, we discuss the connection — inherited from the Hopf fibration between twisted textured strings and skyrmions (or textures). We note that the manner in which the string twists in internal space along the string loop is different from the gauged case: while the gauged case involves a string whose plane of rotation rotates along the loop, the textured string rotates always in the same plane, but with an offset angle increasing along the loop.

We review our conclusions in section V1. Intuition-building examples are developed throughout the text, including the original Alice symmetry-breaking pattern proposed by Schwarz [1], patterns realizable when a larger $S O(6)$ symmetry breaks down, and the BensonManohar-Saadi textured string involving Majorana interactions with point charges. Of these, the first is most testable, as the global symmetry-breaking transition responsible for the nematic phase in liquid crystals; the others are motivated by particle physics GUT phase transitions.

\section{DIVERGENT GLOBAL ALICE STRINGS}

Consider a global symmetry group $G$ broken to a residual subgroup $H$ by some condensate $\langle\phi\rangle$. Divergent strings form under the same topological conditions as gauged strings: whenever the vacuum manifold $G / H$ of degenerate $\langle\phi\rangle$ orientations has nontrivial loops $\pi_{1}(G / H)$. Such loops determine the string's topologically nontrivial boundary conditions: 
that is, the winding of $\langle\phi\rangle$ asymptotically. We parametrize this winding via the angledependent $G$ rotation $U(\varphi)$ which generates it:

$$
\langle\phi(\varphi)\rangle=U(\varphi)\langle\phi\rangle_{o}
$$

Here $U(\varphi)$ acts on $\langle\phi\rangle_{o}$ according to its group representation. Recall that, in the gauged case, $U(\varphi)$ is the Wilson line due to the string's nonabelian flux. Here, it is the same group rotation, inducing the same condensate winding, but with divergent gradient energy cost per unit length.

By the exact sequence for $\pi_{1}(G / H), U(\varphi)$ has two topologically nontrivial forms: it can be a nontrivial loop in $G$, which cannot lie wholly in $H$; or it can be a path in $G$, ending on some element $h_{o}$ in a disconnected component of $H$.

To find an Alice string, which makes some $H$ generators multivalued, we must adopt the second possibility. This is because the symmetries $H$ leaving $\langle\phi(\varphi)\rangle$ invariant, and their generators $T_{h}$, are parallel transported around the string:

$$
\begin{aligned}
& H(\varphi)=U(\varphi) \quad H_{o} U^{-1}(\varphi) \\
& T_{h}(\varphi)=U(\varphi) T_{h, o} U^{-1}(\varphi) .
\end{aligned}
$$

Thus $T_{h}(\varphi)$ becomes multivalued only when a nontrivial $U(2 \pi)$ fails to commute with $T_{h, o}$.

Thus divergent strings are Alice, with multivalued generators $T_{h}(\varphi)$, whenever $U(2 \pi)$ is an element $h_{o}$ which 1) lies in a disconnected component of $H_{o}$, and 2) doesn't commute with Alice generators $T_{h, o}$. We note further that $U(\varphi)$ rotates the condensate, and connects disconnected components of $H$, so that it must be generated, at least partially, by broken symmetry generators $T_{b}$. To minimize string energy, we take $U(\varphi)$ to be generated uniformly by a single broken generator $T_{b}$ :

$$
\begin{aligned}
U(\varphi) & =e^{-i \varphi T_{b}} \\
& =h_{0} \text { at } \varphi=2 \pi .
\end{aligned}
$$

Given this construction of the divergent global Alice string, existence of global monopoles 
formed by twisted string loops is guaranteed by [5]. Charge violation due to Aharonov-Bohm scattering requires more careful analysis. Fundamental fermions $\psi$ carry charges

$$
Q_{h}=\int d^{3} x \psi^{\dagger} T_{h} \psi
$$

and interact with the condensate $\phi$ through effective mass terms of either Dirac or Majorana form:

$$
\mathcal{L}_{\text {int }}= \begin{cases}-m \bar{\psi} \phi \psi+h . c . & \text { Dirac } \\ -m \bar{\psi} \phi \psi_{c}+h . c . & \text { Majorana }\end{cases}
$$

Here the condensate field $\phi$ is in a group representation transforming either as

$$
\phi \rightarrow\left\{\begin{array}{ll}
g \phi g^{\dagger} & \text { Dirac } \\
g \phi g^{T} & \text { Majorana }
\end{array} .\right.
$$

The Dirac mass term is simpler and generalizes readily to charged bosons, so we focus on it here. The Majorana interaction arises in some symmetry breakdowns of $S U(n)$ groups, as for the textured global string model of Benson, Manohar and Saadi. We return to its role in Aharonov-Bohm scattering in section ШD.

For the Dirac-type interaction, the fermion $\psi$ has a spatially varying effective mass matrix

$$
\mathcal{M}=U(\varphi)\langle\phi\rangle_{o} U^{-1}(\varphi)
$$

outside the global string. This determines the fermion's behavior in Aharonov-Bohm scattering around the string. For, under Aharonov-Bohm scattering, a fermion in mass eigenstate $\psi_{a}$ does three things: 1) it tracks the local mass eigenstate $\left.\psi_{a}(\varphi) ; 2\right)$ it acquires the time evolution phase $e^{-i m_{a} t}$; and 3) it acquires a "Berry's phase." This Berry's phase is generally nonabelian, as the residual symmetries $H$ induce fermion mass degeneracies. Thus $\psi_{a}(\varphi)$ mixes with other degenerate eigenstates $\psi_{b}(\varphi)$, according to the nonabelian Wilson line [9]

$$
W(\varphi)=e^{-\int^{\varphi} d \varphi^{\prime} A_{\varphi^{\prime}}}
$$

with the (matrix) gauge field $A_{\varphi^{\prime}}$ given by 


$$
\left(A_{\varphi}\right)_{a b}=\left\langle\psi_{b}(\varphi)\left|\partial_{\varphi}\right| \psi_{a}(\varphi)\right\rangle
$$

in the degenerate subspace. Altogether, then, a fermion $\psi_{a}(\varphi=0)$ becomes, after circumnavigating the string,

$$
\psi_{a}^{\prime}=e^{-i m_{a} t} W(2 \pi) \psi_{a}(2 \pi) .
$$

For the divergent Alice string, the spatially varying mass eigenstates are

$$
\psi_{a}(\varphi)=U(\varphi) \psi_{a}
$$

where $\psi_{a}$ is an eigenstate of the matrix $\langle\phi\rangle_{o}$. Since $U(2 \pi)=h_{o}$, the mass eigenstate $\psi_{a}(\varphi)$ is multivalued whenever $h_{o}$ acts nontrivially on $\psi_{a}$. (Typically, such multivaluedness consists only of multiplying $\psi_{a}$ by a phase - for a $Z_{2}$ Alice string, a minus sign.)

We now consider the nonabelian Berry's phase for our minimal energy divergent Alice string ([I.3). For divergent strings, this Berry's phase is either trivial, or cancels any nonintrinsic multivaluedness in our definition of the mass eigenstate $\psi_{a}(\varphi)$. (For example, setting $\psi_{a}(\varphi)=e^{-i \varphi / 2} \psi_{a}$, an unphysical multivaluedness, induces compensating Berry's phase $W(\varphi)=e^{+i \varphi / 2}$, showing the physical mass eigenstates to be truly constant.) Because the nonabelian Berry's phase will have much richer and more subtle behavior for textured Alice strings, we introduce it carefully here.

For our minimal divergent Alice string ([1.3), equation ([1.9) gives a Berry phase determined by

$$
\left(A_{\varphi}\right)_{a b}=-i\left\langle\psi_{b}(\varphi)\left|T_{b}\right| \psi_{a}(\varphi)\right\rangle=-i\left\langle\psi_{b}\left|T_{b}\right| \psi_{a}\right\rangle
$$

using (II.11). Note that $T_{b}$ is the broken symmetry generator generating $U(\varphi)-$ and thus both string and mass eigenstate winding - while the matrix element is taken between degenerate states.

Mathematically, the broken symmetry generator $T_{b}$ can act on state $\left|\psi_{a}\right\rangle$ in one of three ways. First it can annihilate it, leaving the mass eigenstate (II.11) spatially invariant with no Berry's phase above. Second, and most generically, it can rotate $\left|\psi_{a}\right\rangle$ out of the degenerate 
mass subspace. (Note that unbroken generators rotate $\left|\psi_{a}\right\rangle$ into degenerate states, while broken generators typically rotate $\left|\psi_{a}\right\rangle$ into nondegenerate ones.) In this case, $T_{b}\left|\psi_{a}\right\rangle$ has no component within the degenerate subspace, so the Berry's phase above vanishes. Here the mass eigenstate $\psi_{a}(\varphi)$ does vary spatially, and any apparent multivaluedness is physical. In the third case, the rotated state $T_{b}\left|\psi_{a}\right\rangle$ has components in the degenerate subspace. By basis choice we diagonalize $T_{b}$ within the degenerate subspace, taking $T_{b}\left|\psi_{a}\right\rangle \propto\left|\psi_{a}\right\rangle$ there. This produces an overall numeric phase in Berry's phase $W(\varphi)$, one that exactly cancels an overall numeric phase in $\psi_{a}(\varphi)=U(\varphi) \psi_{a}$. This shows that $T_{b}$ 's action in the degenerate subspace is spurious, causing a nonphysical phase variation in $\psi_{a}(\varphi)$ which is eliminated via the self-consistent Berry's phase approach.

Thus, in circumnavigating the divergent global string, fermion mass eigenstates are always acted on by the effective Wilson loop $W(2 \pi) U(2 \pi)$. Here $U(2 \pi)$ is the Wilson loop for the gauge case. $W(2 \pi)$, the nonabelian Berry's phase, is nontrivial only if the (broken) string generator $T_{b}$ fails to rotate the mass eigenstate entirely out of its degenerate subspace. In that uncommon case, $W(2 \pi)$ cancels any nonphysical phase which creeps into the Wilson loop $U(2 \pi)$.

Behind our calculation of fermion scattering lies the physical question, "Is charge conservation violated in Aharonov-Bohm scattering around the divergent global Alice string?" Details are model-dependent, but the mass eigenstate scattering above lays the foundation for possible charge violation. This is because the effective Wilson loop, $W(2 \pi) U(2 \pi)=$ $W(2 \pi) h_{o}$, makes only some mass eigenstates multivalued, while others remain single-valued. Thus a charge eigenstate

$$
|q\rangle=\sum_{\text {single }} c_{a}\left|\psi_{a}\right\rangle+\sum_{\text {multi }} c_{b}\left|\psi_{b}\right\rangle,
$$

where the first sum ranges over single-valued mass eigenstates, the second over multivalued ones, becomes

$$
|q\rangle^{\prime}=\sum_{\text {single }} c_{a}\left|\psi_{a}\right\rangle+\sum_{\text {multi }} c_{b}^{\prime}\left|\psi_{b}\right\rangle
$$


after circumnavigating the string. Typically, $c_{b}^{\prime}$ differ from $c_{b}$ by a phase. Thus, so long as the charge eigenstate has both single- and multivalued mass eigenstate components, the scattered state is physically distinct, and carries distinct charge, from the initial $|q\rangle$. This means that charges alter, just as in the gauged case, when Aharonov Bohm scattered around the divergent global Alice string. In the usual case where $W(2 \pi)=1$, it is precisely those charges associated with Alice generators $T_{h o}$ — which do not commute with the Wilson loop $U(2 \pi)$ - which may be altered. For in this case, the effective Wilson loop is just the gauge Wilson loop, $U(2 \pi)$, and the Alice charge eigenstates obey

$$
\begin{aligned}
T_{h o}|q\rangle & =q|q\rangle \\
T_{h o}|q\rangle^{\prime}=T_{h o} U(2 \pi)|q\rangle & =q|q\rangle^{\prime}+\left[T_{h o}, U(2 \pi)\right]|q\rangle^{\prime}
\end{aligned}
$$

Thus, in this usual case, all Alice charges not annihilated by $\left[T_{h o}, U(2 \pi)\right]$ are nonconserved in scattering around the divergent global Alice string.

\section{A. The Canonical Divergent Global Alice String}

We illustrate this general formalism with the simplest example, the Schwarz Alice string, whose scattering in the divergent global string case was considered by McGraw. [7] Here $G$ is $S O(3)$, and the Higgs $\phi$ transforms as a matrix and interacts with fundamental fermions through Dirac mass terms. $\phi$ develops the vev

$$
\langle\phi\rangle=\operatorname{diag}(1,1,-2)
$$

with an $O(2)$ residual symmetry $H$ containing z-rotations $R_{z}(\alpha)$ and the discrete symmetry element

$$
h_{o}=R_{x}(\pi)=\operatorname{diag}(1,-1,-1)
$$

$\pi_{1}(G / H)=Z_{2}$ so we have global $Z_{2}$ strings generated by

$$
U(\varphi)=R_{x}(\varphi / 2)
$$


with $U(2 \pi)=h_{o}$. This string is Alice, as $U(2 \pi)$ fails to commute with the unbroken symmetry generator $T_{z}$; in fact, on parallel transport around the string,

$$
T_{z} \rightarrow U(2 \pi) \quad T_{z} \quad U^{-1}(2 \pi)=-T_{z}
$$

We now show that this formal Alice property yields physical Alice behavior — with twisted Alice string loops forming monopoles, and charge-violating Aharonov Bohm scattering. As always for divergent global strings, twisted loops form monopoles due to the arguments of [5]. Charge violation occurs because some fermion mass eigenstates are doublevalued; in particular, we have spatially varying mass eigenstates

$$
\begin{aligned}
& \left\{\left|\psi_{a}(\varphi)\right\rangle\right\}=R_{x}(\varphi / 2)\left\{\left(\begin{array}{l}
1 \\
\end{array}\right),\left(\begin{array}{l} 
\\
1
\end{array}\right),\left(\begin{array}{l} 
\\
1
\end{array}\right)\right\} \\
& =\left\{(1),\left(\begin{array}{c}
\cos (\varphi / 2) \\
\sin (\varphi / 2)
\end{array}\right),\left(\begin{array}{r}
-\sin (\varphi / 2) \\
\cos (\varphi / 2)
\end{array}\right)\right\} .
\end{aligned}
$$

Note that the first two mass eigenstates are degenerate, while the last two are double-valued. The nonabelian Berry's phase vanishes, as the string generator $T_{x}$ does not connect any degenerate mass eigenstates. Thus this example has the same effective Wilson loop $U(2 \pi)$ as in the gauged case, making two of three mass eigenstates double-valued. As expected, fermions reverse charge in circumnavigating the global string, for charge eigenstates

$$
| \pm\rangle=\frac{1}{\sqrt{2}}\left(\begin{array}{r}
1 \\
\pm i
\end{array}\right) \quad|0\rangle=\left(\begin{array}{l} 
\\
1
\end{array}\right)
$$

scatter as $| \pm\rangle \rightarrow|\mp\rangle,|0\rangle \rightarrow-|0\rangle$ in traversing the string. This can be seen directly from the action of the effective Wilson loop $U(2 \pi)=h_{o}$ on the charge eigenstates, or from the underlying spatial variation of the constituent mass eigenstates. 


\section{BUILDING TEXTURED ALICE STRINGS}

Again consider the breaking of a global symmetry $G$ to residual subgroup $H$ by Higgs condensate $\langle\phi\rangle$. Finite tension strings form only if they are textured; that is, if they approach a constant vev $\langle\phi\rangle_{o}$ asymptotically and carry topological winding in their cores. Such winding occurs whenever the vacuum manifold has nontrivial homotopy $\pi_{2}(G / H)$. This describes the condensate's nontrivial variation through degenerate vacuum states in the plane transverse to the string; because each point at spatial infinity approaches the same vacuum state $\langle\phi\rangle_{o}$, the transverse plane becomes identified with a sphere $S^{2}$, and has topologically nontrivial vacuum configurations classified by $\pi_{2}(G / H)$.

We display the condensate's spatial variation in Figure 1, noting that it is generated by some group rotation $U(r, \varphi)$ acting on the single asymptotic vev $\langle\phi\rangle_{o}$ :

$$
\langle\phi(r, \varphi)\rangle=U(r, \varphi)\langle\phi\rangle_{o} .
$$

$U(r, \varphi)$ acts on $\langle\phi\rangle_{o}$ according to its group representation, and varies over $G$ in some singlevalued, nonsingular way. By the exact sequence for $\pi_{2}(G / H), U(r, \varphi)$ has two topologically nontrivial forms: it can be a nontrivial map in $G$, which cannot lie wholly in $H$; or it can be a map in $G$, ending asymptotically on a nontrivial loop $h(\varphi)$ in $H$. Again, to find Alice behavior, we restrict ourselves to the second case, where $U(r, \varphi)$ approaches a nontrivial loop $h(\varphi)$ in $\mathrm{H}$ at large $r$. (Thus $H$ must have nontrivial $\pi_{1}$.) Remarkably, even though the map $U(r, \varphi)$ is single-valued, it can cause charges to become multivalued, or Alice, as we shall see by construction.
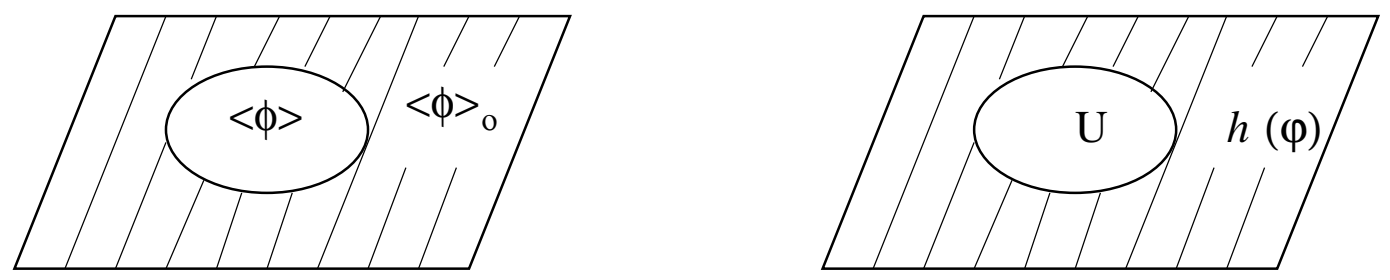

Figure 1: $\quad\langle\phi>$ in $\mathrm{G} / \mathrm{H}$ generated by $\mathrm{U}$ in $\mathrm{G}$; hashed regions show asymptotic behavior. 
Construction could, of course, vary greatly by model; here, however, we suggest a useful ansatz. Say our nontrivial loop $h(\varphi)$ is generated by unbroken generator $T_{h}$, which anticommutes with some group element $b_{o}$. (This happens whenever $G$ contains $S U(2)$ or $S O(3)$ subgroups, but could occur for more exotic symmetry breaking patterns as well.) Taking $b_{o}$ to be generated by broken generator $T_{b}$,

$$
b_{o}=e^{-i F_{o} T_{b}} \quad h(\varphi)=e^{-i \varphi T_{h}},
$$

a nontrivial map $U(r, \varphi)$ is given by

$$
U(r, \varphi)=h(\varphi / 2) e^{-i F(r) T_{b}} h(\varphi / 2) \quad \text { where } \quad F(r) \rightarrow \begin{cases}0 & r \rightarrow \infty \\ F_{o} & r \rightarrow 0\end{cases}
$$

Note that $U=b_{o}$ at the origin, since $b_{o} h=h^{-1} b_{o}$, and approaches the nontrivial loop $h(\varphi)$ at infinite radius. The only caveat is that $U(r, \varphi)$ must be single-valued. This could occur if $h(\pi)$ commutes with $T_{b}$, a possibility we exploit in the canonical Alice model. For our GUTinspired models, however, we insure single-valuedness by taking $T_{b}$ and $T_{h}$ to lay within a partially broken $S U(2)$ subalgebra. We then choose Lie algebra bases so that the generators $T_{b}$ and $T_{h}$ anticommute, with

$$
e^{-i F(r) T_{b}}=\cos F(r) \mathbb{1}-i \sin F(r) T_{b}
$$

within the subspace where $T_{b}$ and $T_{h}$ are nontrivial. (We note that we can make these choices whenever $G$ includes $S U(N)$ factors, for $N \geq 2$, or $S O(N)$ factors, for $N \geq 4$, as we show by construction below). Thus, for these models,

$$
U(r, \varphi)=h(\varphi / 2) e^{-i F(r) T_{b}} h(\varphi / 2)=\cos F(r) h(\varphi)-i \sin F(r) T_{b}
$$

within the subspace where $T_{b}$ and $T_{h}$ are nontrivial. This gives the loop $h(\varphi)$ at $r \rightarrow \infty$, and anticommuting broken group element $b_{o}$ at the origin, where $F=F_{o}=\pi / 2$. Moreover, this map is single-valued and nonsingular everywhere, and so acts on $\langle\phi\rangle_{o}$ to create a nontrivial textured string whenever the loop $h(\varphi)$ is nontrivial in $H$. 
Thus far, our ansatz constructs a nontrivial textured string. When is this string Alice; that is, when does an unbroken generator $T_{h^{\prime}}(r)$ determine non-conserved charge eigenstates in circumnavigating the string? Surprisingly, the answer is nontrivial, and depends on the nonabelian Berry phase to resolve ambiguities. Formally, unbroken generators are parallel transported throughout the textured string:

$$
T_{h}(r, \varphi)=U(r, \varphi) T_{h, o} U^{-1}(r, \varphi) .
$$

Naively, since $U(r, 2 \pi)=U(r, 0)$, all generators are single-valued; none would appear to be Alice. Closer analysis, however, in all our examples below and in section IV, reveals that any generator $T_{h^{\prime}}$ which fails to commute with $U(r, \varphi)$, at any angle $\varphi$, is Alice: its charges alter when Aharonov-Bohm scattered around the string at finite, nonzero radius.

We construct textured Alice strings in three models below, following this ansatz. In the first, a symmetry breakdown of $S O(N)$, the condensate $\langle\phi\rangle$ interacts with charged fermions through a Dirac mass coupling. The second, the Benson, Manohar, Saadi textured string, involves $S U(N)$ symmetry breakdown and Majorana mass couplings. Finally, we construct a textured Alice string in the canonical Schwarz model itself, which exploits subtleties of the embedding of $O(2)$ in $S O(3)$.

\section{A. $S O(N)$ symmetry breakdown and the $S O(6)$ model}

Let the unbroken symmetry group $G$ be $S O(N)$, with Higgs $\phi$ transforming in the adjoint and coupling to fermions $\psi$ through Dirac mass terms (equations (II.6) and (II.5)). For $N \geq 4$, we can construct a textured string varying over an $S O(4)$ subspace, which makes some unbroken generators $T_{h^{\prime}}$ Alice; we show the case with $N=6$ for definiteness.

$\phi$ develops the vev

$$
\langle\phi\rangle=\operatorname{diag}(1,1,1,-1,-1,-1)
$$

with residual symmetry $H=S O(3) \times S O(3) \times Z_{2}$ of rotations in the upper left $3 \times 3$ 
block, rotations in the lower right $3 \times 3$ block, and the discrete element -11 . Now consider the $4 \times 4$ subspace of rows and columns 2 through 5 , where $\phi$ assumes values

$$
\langle\phi\rangle_{\square}=\operatorname{diag}(1,1,-1,-1)
$$

It is in this subspace, of an $S O(4)$ theory broken to $S O(2) \times S O(2)$, where our textured string will vary. We therefore reindex these rows from 1 to 4 in what follows.

A natural basis for so(4) in our subspace is the set $\left\{T_{i j}\right\}$ generating rotations in the six planes $i j$. However we exploit the basis

$$
\begin{array}{ccc}
J_{1}=T_{12}+T_{34} & J_{2}=T_{23}+T_{14} & J_{3}=T_{13}-T_{24} \\
K_{1}=T_{12}-T_{34} & K_{2}=T_{23}-T_{14} & K_{3}=T_{13}+T_{24}
\end{array}
$$

which makes the isomorphism $S O(4)=S U(2) \times S U(2)$ clear:

$$
\left[J_{i}, J_{j}\right]=2 i \epsilon_{i j k} J_{k} \quad\left[K_{i}, K_{j}\right]=2 i \epsilon_{i j k} K_{k} \quad\left[J_{i}, K_{j}\right]=0
$$

Moreover, the sets $\left\{J_{i}\right\}$ and $\left\{K_{i}\right\}$ truly behave like Pauli matrices: anticommuting among themselves, and squaring to $\mathbb{1}$ within the 4-dimensional subspace, so that

$$
e^{-i \alpha J_{i}}=\cos \alpha 1-i \sin \alpha J_{i}
$$

within the $\square$-subspace.

Now consider the so(3) subalgebra of $g$ generated by $\left\{J_{i}\right\}$. Only $J_{1}$ remains unbroken by the vev $\langle\phi\rangle_{\square}$; it generates the nontrivial loop

$$
h(\varphi)=e^{-i \varphi J_{1}}
$$

in our full unbroken symmetry group $S O(3) \times S O(3) \times Z_{2}$. Since $J_{2}$ anticommutes with $J_{1}$, the map

$$
U(r, \varphi)=h(\varphi / 2) e^{-i F(r) J_{2}} h(\varphi / 2)=\cos F(r) h(\varphi)-i \sin F(r) J_{2}
$$

in the $S O(4)$ subspace, with 


$$
F(r) \rightarrow \begin{cases}0 & r \rightarrow \infty \\ \pi / 2 & r \rightarrow 0\end{cases}
$$

acts on $\langle\phi\rangle_{\square}$ to create a nontrivial textured string. This is because $U(r, \varphi)$ is single-valued and nonsingular, giving $b_{o}=-i J_{2}$ at the origin and the nontrivial loop $h(\varphi)$ asymptotically.

Because $\phi$ transforms in the adjoint, the textured string looks like

$$
\langle\phi\rangle(r, \varphi)=U(r, \varphi)\langle\phi\rangle_{o} U^{-1}(r, \varphi)
$$

We can also use the unbroken symmetry to rewrite this as

$$
\langle\phi\rangle(r, \varphi)=h(\varphi / 2) e^{-i F(r) J_{2}}\langle\phi\rangle_{o} e^{+i F(r) J_{2}} h^{-1}(\varphi / 2)
$$

We see here how ambiguities arise in establishing the string as Alice. For the first form (III.15) for $\langle\phi\rangle_{\square}$ encourages us to defines spatially varying mass eigenstates as

$$
\psi_{a}(r, \varphi)=U(r, \varphi) \psi_{a}
$$

where $\psi_{a}$ are eigenstates of $\langle\phi\rangle_{o}$. These would be single-valued, implying that no charges are Alice. However, the second form indicates spatially varying mass eigenstates of

$$
\psi_{a}(r, \varphi)=h(\varphi / 2) e^{-i F(r) J_{2}} \psi_{a}
$$

double-valued for all $\psi_{a}$ in the four-dimensional $\square$-subspace (and single-valued elsewhere, where $h(\varphi / 2)=\mathbb{1})$. Thus any charge eigenstates with components straddling the $\square$ and non- $\square$ subspaces would appear to be Alice. Careful nonabelian Aharonov-Bohm scattering analysis resolves this ambiguity in section $\mathbb{I V}$, with a physically sensible answer. At the origin (where any angle-dependence would be singular) and at spatial infinity (where the textured string leaves no physical trace, since the vev $\langle\phi\rangle$ is constant), no charges are Alice. However, at finite intermediate $r$, all charges whose generators $T_{h^{\prime}}$ fail to commute with $U(r, \varphi)$ become multivalued, or Alice, in a curiously $r$-dependent way. 


\section{B. $S U(N) \rightarrow S O(N)$; the Benson-Manohar-Saadi string}

We here review the Benson-Manohar-Saadi string in simplified formalism. Let the unbroken symmetry group $G$ be $S U(N)$, with Higgs $\phi$ transforming as

$$
\phi \rightarrow g \phi g^{T}
$$

and coupling to fermions $\psi$ through the Majorana mass term ([1.5). For $N \geq 2$, there exists a textured string varying over an $S U(2)$ subspace, which makes some unbroken generators $T_{h^{\prime}}$ Alice; we show the case with $N=3$ for definiteness.

Here $\phi$ develops the vev

$$
\langle\phi\rangle=1
$$

with residual symmetry $H=S O(3)$ of orthogonal rotations $\left(h^{T}=h^{-1}\right)$. We take the $S U(3)$ generators to be

$$
\begin{array}{ll}
\text { symmetric: } & \sigma_{x(12)}, \sigma_{x(23)}, \sigma_{x(31)}, \sigma_{z(12)}, \frac{1}{\sqrt{3}} \operatorname{diag}(1,1,-2) \\
\text { antisymmetric: } & \sigma_{y(12)}, \sigma_{y(23)}, \sigma_{y(31)}
\end{array}
$$

where $\sigma_{x(i j)}, \sigma_{y(i j)}, \sigma_{z(i j)}$ denote Pauli matrices in the $(i j)$ plane. Under symmetry-breaking, all symmetric generators are broken, all antisymmetric generators unbroken. (This statement generalizes to all $N \geq 3$, with unbroken subgroup $S O(N)$.)

We now construct the textured string in the (12) $S U(2)$ subgroup. Here the unbroken generator $\sigma_{y(12)}$ generates the nontrivial loop

$$
h(\varphi)=e^{-i \varphi \sigma_{y}(12)}
$$

and the broken generator $\sigma_{z(12)}$ both anticommutes with $\sigma_{y(12)}$ and obeys

$$
e^{-i \alpha \sigma_{z(12)}}=\cos \alpha \mathbb{1}-i \sin \alpha \sigma_{z(12)}
$$

in the (12) plane. Thus our ansatz gives a nontrivial textured string generated by

$$
U(r, \varphi)=h(\varphi / 2) e^{-i F(r) \sigma_{z(12)}} h(\varphi / 2)=\cos F(r) h(\varphi)-i \sin F(r) \sigma_{z(12)}
$$


in the (12) plane, with

$$
F(r) \rightarrow \begin{cases}0 & r \rightarrow \infty \\ \pi / 2 & r \rightarrow 0\end{cases}
$$

Again $U(r, \varphi)$ is single-valued and nonsingular, giving $b_{o}=-i \sigma_{z(12)}$ at the origin and the nontrivial loop $h(\varphi)$ asymptotically.

Applying the transformation law ([III.19), the textured string looks like

$$
\langle\phi\rangle(r, \varphi)=U(r, \varphi)\langle\phi\rangle_{o} U^{T}(r, \varphi)=h(\varphi / 2) e^{-2 i F(r) \sigma_{z(12)}} h^{-1}(\varphi / 2)
$$

Again, the existence of double-valued mass eigenstates in the (12) plane,

$$
\psi_{ \pm}(\varphi)=h(\varphi / 2)\left\{(1),\left(\begin{array}{l} 
\\
1
\end{array}\right)\right\}=\left\{\left(\begin{array}{c}
\cos (\varphi / 2) \\
\sin (\varphi / 2)
\end{array}\right),\left(\begin{array}{r}
-\sin (\varphi / 2) \\
\cos (\varphi / 2)
\end{array}\right)\right\}
$$

makes all (13) and (23) unbroken generators appear to be Alice (since the mass eigenstate $(0,0,1)$ is spatially invariant). But such omnipresent Alice behavior again cannot be physical

- our solution is nonsingular at the origin and constant at spatial infinity, and thus cannot physically cause Alice scattering at those radii.

Again we resolve the conundrum in section [V], via careful nonabelian Aharonov-Bohm scattering analysis. At the origin and at spatial infinity, we show that no charges are Alice. However, at finite intermediate $r$, all charges whose generators $T_{h^{\prime}}$ fail to commute with $U(r, \varphi)$ show $r$-dependent Alice behavior.

\section{The Canonical Alice model}

Surprisingly, the rich laboratory of the original Schwarz model (or nematic liquid crystal) also supports a textured Alice string given by our ansatz. Here, from section [IA, $G$ is $S O(3)$, with the Higgs $\phi$ transforming as a matrix and giving Dirac masses to the fundamental fermions. $\phi$ develops the vev

$$
\langle\phi\rangle=\operatorname{diag}(1,1,-2)
$$


with an $O(2)$ residual symmetry $H$ containing z-rotations $R_{z}(\alpha)$ and the discrete symmetry element

$$
h_{o}=R_{x}(\pi)=\operatorname{diag}(1,-1,-1)
$$

which anticommutes with unbroken generator $T_{z}$.

A topological subtlety arises here. From the exact sequence for $\pi_{2}(G / H)$, all nontrivial textured strings are generated by a map $U(r, \varphi)$ ending on a nontrivial loop $h(\varphi)$ in $H$. Such a map $U(r, \varphi)$ unwinds the loop in $H$ in the larger group $G$. For the symmetry breaking at hand, we note that only $O(2)$ loops of even winding are unwindable in $S O(3)$. Thus, the lowest winding textured string must end on a winding 2 loop in $O(2)$,

$$
h(\varphi)=R_{z}(2 \varphi)
$$

Given this, our ansatz gives a lowest winding textured string, with

$$
U(r, \varphi)=R_{z}(\varphi) \quad R_{x}(F(r)) \quad R_{z}(\varphi) \quad \text { where } F(r) \rightarrow \begin{cases}0 & r \rightarrow \infty \\ \pi & r \rightarrow 0\end{cases}
$$

Note that $U$ is manifestly single-valued. It approaches the nontrivial loop $R_{z}(2 \varphi)$ at infinite radius, and the value $R_{x}(\pi)=h_{o}$ at the origin, since $h_{o} R_{z}(\varphi)=R_{z}^{-1}(\varphi) h_{o}$.

$U$ determines the textured string

$$
\langle\phi\rangle(r, \varphi)=U(r, \varphi)\langle\phi\rangle_{o} U^{-1}(r, \varphi)=R_{z}(\varphi) R_{x}(F(r))\langle\phi\rangle_{o} R_{x}^{-1}(F(r)) R_{z}^{-1}(\varphi) .
$$

This determines apparently single-valued mass eigenstates,

$$
\left\{\psi_{a}(\varphi)\right\}=R_{z}(\varphi) R_{x}(F(r))\left\{\left(\begin{array}{l}
1 \\
1
\end{array}\right),\left(\begin{array}{l} 
\\
1
\end{array}\right)\right\}
$$

However, degeneracy of the first two eigenstates again forces a careful nonabelian AharonovBohm analysis, which again reveals nontrivial Alice behavior at finite radius. 


\section{CHARGE SCATTERING BY TEXTURED ALICE STRINGS}

We have identified three models with textured strings. Each string is generated by the action on a vev $\langle\phi\rangle_{o}$ of a single-valued group map

$$
U(r, \varphi)=h(\varphi / 2) e^{-i F(r) T_{b}} \quad h(\varphi / 2) \quad \text { where } \quad F(r) \rightarrow \begin{cases}0 & r \rightarrow \infty \\ F_{o} & r \rightarrow 0\end{cases}
$$

with $h(\varphi)=e^{-i \varphi T_{h}}$ a minimal nontrivial loop in $H$ shrinkable in $G$.

We now consider Aharonov-Bohm scattering of fundamental fermions $\psi$ by such a string. Details of the scattering analysis differ, for Dirac and Majorana fermions; however, both give the same result: charge-violating scattering at finite nonzero radii only. We treat each in turn.

\section{A. For Dirac Fermions}

When $\psi$ couples to $\phi$ through Dirac couplings (II.5), $\phi$ transforms under $U$ by conjugation and produces the effective mass matrix

$$
\langle\phi\rangle(r, \varphi)=h(\varphi / 2) e^{-i F(r) T_{b}}\langle\phi\rangle_{o} e^{+i F(r) T_{b}} h^{-1}(\varphi / 2)
$$

for $\psi$. Choosing $\langle\phi\rangle_{o}$ diagonal (as in our examples) gives apparent spatially varying mass eigenstates

$$
\psi_{a}(r, \varphi)=h(\varphi / 2) e^{-i F(r) T_{b}} \hat{e}_{a}
$$

with $\hat{e}_{a}$ a unit basis vector. These mass eigenstates may or may not appear multivalued.

First, $T_{h}$ may annihilate $e^{-i F(r) T_{b}} \hat{e}_{a}$, giving an angle-independent mass eigenstate $\psi_{a}$. Otherwise, there are two possibilities for $\psi_{a}$. If the unit winding loop in $H$ is shrinkable in $G$ (as for the $S O(6)$ example of section III A), the remaining mass eigenstates are double-valued. However, if the smallest winding loop in $H$ shrinkable in $G$ has even winding (as for the canonical Alice model example of section [IIC), all mass eigenstates are single-valued. 
However, the relevant object, as discussed for divergent global strings in section [1], is not the mass eigenstate $(\mathbb{R . 3})$ but the mass eigenstate acted on by nonabelian Berry's phase $W(r, \varphi)$ :

$$
W(r, \varphi)=e^{-\int^{\varphi} d \varphi^{\prime} A_{\varphi^{\prime}}}
$$

where

$$
\left(A_{\varphi}\right)_{a b}=\left\langle\psi_{b}(r, \varphi)\left|\partial_{\varphi}\right| \psi_{a}(r, \varphi)\right\rangle
$$

within a subspace of degenerate mass eigenstates $\psi_{a}(r, \varphi)$. For our mass eigenstates (IV.3), this is determined by

$$
\begin{aligned}
& \left(A_{\varphi}\right)_{a b}=\frac{-i}{2}\left\langle e_{b}\left|e^{+i F(r) T_{b}} T_{h} e^{-i F(r) T_{b}}\right| e_{a}\right\rangle \\
& =\left\langle e_{b}\left|\left(\cos [k F(r)]\left(-i T_{h} / 2\right)+\sin [k F(r)]\left(-i T_{b^{\prime}} / 2\right)\right)\right| e_{a}\right\rangle .
\end{aligned}
$$

Here $T_{b^{\prime}}$ is another broken generator $\left(J_{3}\right.$ for the $S O(6)$ case, $T_{y}$ for the canonical Alice case); $k$ is 2 for the $S O(6)$ case and 1 for the canonical Alice case. This simple reduction comes from special algebraic features of the groups chosen: for $S O(6)$, from the properties discussed in equations (III.10) through (III.11); for the canonical case, from traits of the rotation group $S O(3)$ and its generators. In both cases, the broken generator $T_{b^{\prime}}$ does not connect degenerate mass eigenstates, while the unbroken generator connects only degenerate states. Thus the nonabelian Berry's phase acts on mass eigenstates as

$$
W(r, \varphi)=e^{+i \varphi \cos (k F(r)) T_{h} / 2} .
$$

Thus, in scattering around the string, mass eigenstates $\psi_{a}(r, \varphi=0)=e^{-i F(r) T_{b}} \hat{e}_{a}$ are acted on by an effective Wilson loop

$$
W(r, 2 \pi) h(\pi)=h(\pi[1-\cos (k F(r))]) .
$$

This gives single-valued eigenstates at spatial infinity, where $F=0$, and at the origin, where $k F=\pi$ for both models. For finite $r$, however, all mass eigenstates not annihilated by $T_{h}$ 
become multivalued. Thus we have multivalued mass eigenstates (for the $S O(6)$ model, those induced by $\hat{e}_{a}$ in the $\square$-subspace; for the canonical Alice model, those induced by $\hat{e}_{1}$ and $\hat{e}_{2}$ ); and we have single-valued mass eigenstates (for the $S O(6)$ model, those induced by $\hat{e}_{a}$ outside the $\square$-subspace; for the canonical Alice model, the one induced by $\left.\hat{e}_{3}\right)$. As discussed in the last paragraph preceding section $1 \mathrm{~A}$, this means that charge eigenstates with both multi- and single-valued components typically undergo charge-violating Aharonov-Bohm scattering.

More specifically, consider the angle $\alpha(r) \equiv \pi[1-\cos (k F(r))] . \quad h(\alpha(r))$ truly acts as an r-dependent Wilson loop, for Aharonov-Bohm scattering around the textured Alice string. To see consequences of this Wilson loop, we consider two specific Aharonov-Bohm scattering experiments: in the first, we bring a charge radially inward from spatial infinity to $r$, circumnavigate the string, then return radially to spatial infinity to compare or interfere with untransported asymptotic charges. In the second, an observer within the texture defines charges, as related to the unbroken symmetries he observes locally, and observes parallel transport of those charges around the string. Either case gives the same criterion for apparent charge violation, and in each, this criterion is met generically for circumnavigation at finite nonzero $r$.

\section{Reference Charges at Infinity}

First consider comparison of charges defined at spatial infinity. Here we define charge eigenstates $\left|q_{h^{\prime}}\right\rangle$ for all unbroken generators $T_{h^{\prime}}$. We note first that the nonabelian Aharonov Bohm gauge field $A_{r}$ (analogous to equation (IV.5)) vanishes for our models. Thus radial paths contribute no Aharonov-Bohm phase, and mass eigenstates simply track the $r$-dependent eigenstate ([D.3) during any radial transit. Thus, on the radial transit inward, the charge eigenstate $\left|q_{h^{\prime}}\right\rangle$ of generator $T_{h^{\prime}}$ evolves to

$$
\left|q_{h^{\prime}}\right\rangle_{\text {at } r}=e^{-i F(r) T_{b}} \quad\left|q_{h^{\prime}}\right\rangle,
$$


taking $\varphi=0$. After parallel transport around the string, the charge eigenstate acquires the effective Wilson loop $h(\alpha(r))$ :

$$
\left|q_{h^{\prime}}\right\rangle_{\text {at } r}^{\prime}=h(\alpha(r)) e^{-i F(r) T_{b}}\left|q_{h^{\prime}}\right\rangle
$$

Now this circumnavigated charge must be transported back to spatial infinity, for comparison with other asymptotic charges. This gives for the final transported charge

$$
\left|q_{h^{\prime}}\right\rangle_{\text {at } \infty}^{\prime}=e^{+i F(r) T_{b}} \quad h(\alpha(r)) e^{-i F(r) T_{b}}\left|q_{h^{\prime}}\right\rangle
$$

Note we can see this by decomposing our circumnavigated charge ([V.10) at $r$ into mass eigenstates $e^{-i F(r) T_{b}} \quad\left|e_{a}\right\rangle$ at $r$ :

$$
\left|q_{h^{\prime}}\right\rangle_{\text {at } r}^{\prime}=\sum_{a}\left(e^{-i F(r) T_{b}}\left|e_{a}\right\rangle\right)\left\langle e_{a}\left|e^{+i F(r) T_{b}} h(\alpha(r)) e^{-i F(r) T_{b}}\right| q_{h^{\prime}}\right\rangle
$$

Each mass eigenstate $e^{-i F(r) T_{b}}\left|e_{a}\right\rangle$ then adiabatically tracks back to its value $\left|e_{a}\right\rangle$ at $r=\infty$, giving

$$
\begin{aligned}
\left|q_{h^{\prime}}\right\rangle_{\text {at } \infty}^{\prime} & =\sum_{a}\left|e_{a}\right\rangle\left\langle e_{a}\left|e^{+i F(r) T_{b}} h(\alpha(r)) e^{-i F(r) T_{b}}\right| q_{h^{\prime}}\right\rangle \\
& =e^{+i F(r) T_{b}} h(\alpha(r)) e^{-i F(r) T_{b}}\left|q_{h^{\prime}}\right\rangle .
\end{aligned}
$$

Thus, asymptotic symmetry generators $T_{h^{\prime}}$ define charge eigenstates $\left|q_{h^{\prime}}\right\rangle$ which acquire the effective Wilson loop $e^{+i F(r) T_{b}} h(\alpha(r)) e^{-i F(r) T_{b}}$ in our thought experiment: when brought in to radius $r$, parallel transported about the string, then returned to spatial infinity.

\section{Reference Charges at $r$}

Now consider charges as defined by an observer within the texture, at radius $r$ and angle $\varphi=0$. Because $\langle\phi\rangle$ assumes a nonasymptotic value at finite $r$, its residual symmetry group $H$ is not the asymptotic one. In particular, for our $\langle\phi\rangle$-solutions (IV.2), the unbroken symmetry generators leaving $\langle\phi\rangle$ invariant at $r$ are

$$
T_{h^{\prime}, r}=e^{-i F(r) T_{b}} \quad T_{h^{\prime}} e^{+i F(r) T_{b}}
$$


again for $\varphi=0$. Thus the charge eigenstates at $r$ are given by

$$
\left|q_{h^{\prime}}\right\rangle_{\text {at } r}=e^{-i F(r) T_{b}}\left|q_{h^{\prime}}\right\rangle,
$$

related to the asymptotic charges by change of basis. Note that this is the same equation as equation (IV.9) for asymptotic charge eigenstates parallel transported to $r$; however, here the meaning is different, as these are the charge eigenstates as defined at $r$.

After parallel transport around the string, the charge eigenstate acquires the effective Wilson loop $h(\alpha(r))$ :

$$
\left|q_{h^{\prime}}\right\rangle_{\text {at } r}^{\prime}=h(\alpha(r)) e^{-i F(r) T_{b}}\left|q_{h^{\prime}}\right\rangle
$$

Thus, for an observer within the texture, symmetry generators $e^{-i F(r) T_{b}} T_{h^{\prime}} e^{+i F(r) T_{b}}$ define charge eigenstates $e^{-i F(r) T_{b}}\left|q_{h^{\prime}}\right\rangle$, which acquire the Wilson loop $h(\alpha(r))$ when parallel transported about the string.

We thus see that our two experiments, involving asymptotic or $r$-defined charge, are related by change of basis, and give the same criterion for charge nonconservation. Recall that any charge $q_{h^{\prime}}$ is nonconserved if 1) its associated generator fails to commute with the Wilson loop; and 2) the charge eigenstate is not annihilated by the nontrivial commutator, as discussed in equation ([I.15). Here that means charge $q_{h^{\prime}}$ will be nonconserved, in either thought experiment, if

$$
\left[T_{h^{\prime}}, e^{+i F(r) T_{b}} h(\alpha(r)) e^{-i F(r) T_{b}}\right]\left|q_{h^{\prime}}\right\rangle \neq 0
$$

or equivalently, for finite nonzero $r$,

$$
\left[T_{h^{\prime}}, e^{+i F(r) T_{b}} T_{h} e^{-i F(r) T_{b}}\right]\left|q_{h^{\prime}}\right\rangle \neq 0
$$

Recalling the algebraic result (IV.6) for our models, our criterion for $q_{h^{\prime}}$ charge violation becomes

$$
\left[T_{h^{\prime}},\left(\cos [k F(r)] T_{h}+\sin [k F(r)] T_{b^{\prime}}\right)\right]\left|q_{h^{\prime}}\right\rangle \neq 0
$$


with $k$ and $T_{b^{\prime}}$ as given after equation ([V.6). That is, in each model, the textured string varies only over some $S U(2)$ (or $S O(3))$ subgroup of $G$, in which $U(r, \varphi)$ assumes nontrivial values. Unless the generator $T_{h^{\prime}}$ commutes with that entire subgroup — thus being entirely insensitive to the string $-T_{h^{\prime}}$ will show Alice behavior, through charge violation at finite nonzero radius. For the canonical Alice/nematic liquid crystal model of section П119, this means that charge associated with the single unbroken generator $T_{h}=T_{h^{\prime}}=T_{z}$ is violated at all finite nonzero $r$. For the $S O(6)$ model of section III A, we have six unbroken generators $T_{12}, T_{13}, J_{1}, K_{1}, T_{46}, T_{56}$ with $T_{h}=J_{1}=T_{23}+T_{45}$. All charges are violated at finite nonzero radii, except the one associated with $K_{1}$. This charge must be conserved: in our $S O(4)$ subgroup's $S U(2) \times S U(2)$ product structure, the textured Alice string varies over the $J$ associated $S U(2)$ and commutes with the $K$-associated one.

The details of this charge violation are more subtle than the divergent case, however; they consist not of a simple $Z_{2}$ charge flip $| \pm\rangle \rightarrow|\mp\rangle$. Instead an r-dependent rotation mixes, say, a positively charged eigenstate into a linear combination of positive, negative, and neutral eigenstates. For example, in our $S O(6)$ model of section $\Pi 11 \mathrm{~A}$, the unbroken generator $T_{h^{\prime}}=T_{12}$ has eigenstates which undergo charge-violating Aharonov Bohm scattering at finite $r$. The $T_{12}$ charge eigenstates $| \pm\rangle$ scatter to

$$
| \pm\rangle^{\prime}=\frac{1}{2}(1+\cos \alpha)| \pm\rangle+\frac{1}{2}(1-\cos \alpha)|\mp\rangle \pm \frac{i}{\sqrt{2}} \sin \alpha|0\rangle
$$

using the effective Wilson loop discussed above; where $|0\rangle$ is the $r$-dependent neutral charge eigenstate

$$
|0\rangle=\cos (k F)\left|e_{3}\right\rangle+\sin (k F)\left|e_{4}\right\rangle
$$

Thus scattered charge remains unchanged at the origin and spatial infinity; elsewhere, it becomes a radially dependent admixture of variously charged states. Note that, although this textured Alice string is a $Z_{2}$ string topologically, its charge violation is generally not $Z_{2}$ : two traversals around the string are not trivial, at most radii. Only at the special "midpoint" radius, where $\alpha=\pi, k F=\pi / 2$, does a $Z_{2}$ scattering occur: $| \pm\rangle \rightarrow|\mp\rangle$. 
Similarly, for our canonical Alice/nematic liquid textured Alice string of section IIIC, $| \pm\rangle$ charge eigenstates of $T_{h}=T_{z}$ scatter to

$$
\begin{aligned}
| \pm\rangle^{\prime}= & \frac{1}{2}\left[2 \cos (2 \alpha)+(1-\cos (2 \alpha)) \sin ^{2} F\right]| \pm\rangle-\frac{1}{2}(1-\cos (2 \alpha)) \sin ^{2} F|\mp\rangle \\
& +\frac{1}{\sqrt{2}} \sin F[-\sin (2 \alpha) \pm i \cos F(1-\cos (2 \alpha))]|0\rangle
\end{aligned}
$$

Again we find nontrivial, but non- $Z_{2}$, mixing of the charge eigenstate into states of different charge. At the string "midpoint," $\alpha=\pi, F=\pi / 2$, the charge is actually conserved, as $| \pm\rangle \rightarrow| \pm\rangle$. Elsewhere, however, for $0<F<\pi$ (finite nonzero $r$ ), the charge eigenstates mix nontrivially.

We have thus shown that circumnavigation of the string at finite radius violates charge conservation, for charges defined either asymptotically or locally, in accordance with local unbroken symmetries. Those charges associated with unbroken symmetries are simply nonconserved, at finite radius.

In the gauged or divergent global Alice string case, charge violation occurs because local residual symmetries $h$ cannot be consistently extended to singlevalued symmetries $h(\varphi)$. Here that is not true - the fact that the group map $U(r, \varphi)$ generating the string is single-valued induces perfectly single-valued symmetries, and symmetry generators $T_{h^{\prime}}$, when parallel transported around the string by (III.2). However, no set of associated conserved charges can be defined, at finite radius. One might hope some "hidden" set of charge eigenstates, murkily connected with the local unbroken symmetry, might emerge. However, the only candidates for such conserved charge eigenstates are those simultaneous charge eigenstates $\left|q_{h}, q_{h^{\prime}}, \ldots\right\rangle$, for all $T_{h^{\prime}}$ commuting with $T_{h}$. These, at least, are conserved in circumnavigating the string, acquiring only the r-dependent anyonic phase $e^{-i q_{h} \alpha}$. However, any deviation from a circular path in traversing the string violates these eigenstate's charge, for the same reason that asymptotically defined charge becomes violated at finite radius. That is, charge is nonconserved because radial transit causes the charge eigenstates to change and become misaligned with the Wilson loop $h(\alpha)$. 


\section{For Majorana Fermions}

Understanding the Aharonov Bohm scattering for Majorana fermions in the BensonManohar-Saadi model is slightly more complex. Here fermions $\psi$ interact with the textured string

$$
\langle\phi\rangle(r, \varphi)=U(r, \varphi)\langle\phi\rangle_{o} U^{T}(r, \varphi)=h(\varphi / 2) e^{-2 i F(r) \sigma_{z(12)}} h^{-1}(\varphi / 2)
$$

via the mass interaction $-m \bar{\psi} \phi \psi_{c}+$ h.c. At first glance, this gives the mass eigenstates (【II.27), with mass eigenvalues $m e^{\mp 2 i F(r)}$, and with nonabelian Aharonov Bohm phase cancelling the apparent angular variation of the mass eigenstates. However, to get the masses and nonabelian Berry's phase correct, we must look more closely at the bispinor $\left(\psi, \psi_{c}\right)$ and how it obtains its mass. We include a self-conjugate bare Majorana mass matrix $M$ for clarity. The full mass matrix is

$$
\left(\begin{array}{cc} 
& M+m\langle\phi\rangle \\
M+m\langle\phi\rangle^{\dagger} &
\end{array}\right)
$$

acting on $\left(\psi, \psi_{c}\right)$. It has mass eigenstates

$$
\psi_{1 \pm}=\frac{1}{\sqrt{2 \omega * \omega}}\left(\begin{array}{c}
\omega \psi_{+} \chi \\
\pm \omega^{*} \psi_{+} \chi
\end{array}\right), \psi_{2 \pm}=\frac{1}{\sqrt{2 \omega * \omega}}\left(\begin{array}{c}
\omega^{*} \psi_{-} \chi \\
\pm \omega \psi_{-} \chi
\end{array}\right)
$$

where

$$
\omega=\left(M+m e^{-2 i F}\right)^{1 / 2}
$$

$\psi_{ \pm}$are the mass eigenstates ([II.27) in flavor space and $\chi$ is the self-conjugate field $\psi+\psi_{c}$ in spin space. These have associated, spatially varying mass eigenvalues

$$
\lambda_{ \pm}= \pm \omega^{*} \omega= \pm \sqrt{M^{2}+m^{2}+2 M m \cos (2 F)}
$$

with degeneracies between $\psi_{1+}$ and $\psi_{2+}, \psi_{1-}$ and $\psi_{2-}$.

We now calculate the nonabelian Aharonov Bohm phase (IV.5) due to these degeneracies. First note that $\partial_{\varphi}$ acting on any of the spatially varying mass eigenstates (IV.25) is just 
$\left(-i \sigma_{y(12)} / 2,-i \sigma_{y(12)} / 2\right)$ acting on that eigenstate, as the $\varphi$-variation of each eigenstate is simply the $h(\varphi / 2)$ prefactor inherited from flavor mass eigenstates (III.27). And $i \sigma_{y(12)}$ acts on the flavor mass eigenstates (III.27) by sending $\psi_{-} \rightarrow \psi_{+}, \psi_{+} \rightarrow-\psi_{-}$. This affects the full mass eigenstates (IV.25) as follows:

$$
\left(i \sigma_{y(12)}, i \sigma_{y(12)}\right): \psi_{a \pm} \rightarrow-\epsilon_{a b 3} \psi_{b \pm}^{*}
$$

Thus our nonabelian Aharonov Bohm gauge field $A_{\varphi}$ mixes only degenerate mass eigenstates, with matrix elements

$$
\begin{aligned}
\left(A_{\varphi}\right)_{a b, \pm} & =\left\langle\psi_{b \pm}(r, \varphi)\left|\partial_{\varphi}\right| \psi_{a \pm}(r, \varphi)\right\rangle=\frac{1}{2} \epsilon_{a b 3}\left\langle\psi_{b \pm} \mid \psi_{b \pm}^{*}\right\rangle \\
& =\frac{1}{4 \omega^{*} \omega} \epsilon_{a b 3}\left(\omega^{2}+\omega^{* 2}\right) \\
& =\epsilon_{a b 3} \frac{M+m \cos (2 F)}{2 \sqrt{M^{2}+m^{2}+2 M m \cos (2 F)}}
\end{aligned}
$$

This gives for the nonabelian Wilson line

$$
W(r, \varphi)=h^{-1}\left(\frac{M+m \cos (2 F)}{2 \sqrt{M^{2}+m^{2}+2 M m \cos (2 F)}} \varphi\right),
$$

where $h(\varphi)$ is now understood as the direct product $\left(e^{-i \varphi \sigma_{y(12)}}, e^{-i \varphi \sigma_{y(12)}}\right)$ acting on the bispinor $\left(\psi, \psi_{c}\right)$. Thus the $\varphi=0$ mass eigenstates (IV.25) are acted on by

$$
h(\pi) W(r, 2 \pi)=h\left(\left(1-\frac{M+m \cos (2 F)}{\sqrt{M^{2}+m^{2}+2 M m \cos (2 F)}}\right) \pi\right)
$$

in traversing the string. This gives single-valued mass eigenstates at spatial infinity and the origin, where $F$ assumes the values 0 and $\pi / 2$ respectively. Elsewhere, mass eigenstates become multivalued in traversing the string. In the case where the bare Majorana mass $M$ is zero, our effective Wilson loop is

$$
h(\pi) W(r, 2 \pi)=h((1-\cos (2 F)) \pi) .
$$

We note that this is identical to the effective $r$-dependent Wilson loop $h(\alpha)$ we obtained for the Dirac case above. And identical results ensue. Note that, as in the Dirac case, local symmetry generators vary radially: 


$$
T_{h^{\prime}, r}=e^{-i F(r) \sigma_{z(12)}} T_{h^{\prime}} e^{+i F(r) \sigma_{z(12)}}
$$

due to the symmetry transformation rule $\langle\phi\rangle \rightarrow g\langle\phi\rangle g^{T}$. The mismatch - that is, noncommutativity - between the Wilson loop $h(\alpha)$, generated by $T_{h}$, and the radially twisted local unbroken generators $T_{h^{\prime}, r}$, is why a local observer at finite $r$ perceives charge violation, when charge associated with unbroken symmetries at $r$ circumnavigate the string. Similarly, the full mass eigenstates (IV.25) vary radially. For $M=0$, in fact, they vary as if acted on by the flavor space operator $\left(e^{-i F(r) \sigma_{z(12)}}, e^{+i F(r) \sigma_{z(12)}}\right)$. This causes the same noncommutativity problem as in the Dirac case, when asymptotic charges approach finite radius, tracking radially varying mass eigenstates; then circumnavigate the string, acquiring the mismatched Wilson loop $h(\alpha)$; then return to spatial infinity. As in the Dirac case, for either thought experiment - circumnavigation of the string at finite radius by charges defined either locally, or brought in from infinity - charges associated with the asymptotic generator $T_{h^{\prime}}$ are violated whenever

$$
\left[T_{h^{\prime}},\left(\cos [2 F(r)] \sigma_{y(12)}+\sin [k F(r)] \sigma_{x(12)}\right)\right]\left|q_{h^{\prime}}\right\rangle \neq 0
$$

following analysis similar to that preceding equation (IV.19) for the Dirac case. This means in particular, for the $N=3$ BMS string of section ШIIB, that all charges are violated at finite radius.

\section{SKYRMIONS AS TWISTED TEXTURED ALICE STRINGS}

We now show that the textured Alice strings constructed here can be twisted to form topologically nontrivial skyrmions - that is, point defects with nontrivial $\pi_{3}(G / H)$. The twist we consider is the following: bend the string into a loop, with angle $\theta$ along the string core $2 \pi$-periodic. As $\theta$ increases along the loop, twist the textured string by starting its $h$-rotation at increasing offset angle $\varphi_{o}=\theta$ : 
$U(r, \theta+\varphi)=h((\varphi+\theta) / 2) e^{-i F(r) T_{b}} h((\varphi+\theta) / 2) \quad$ where $\quad F(r) \rightarrow \begin{cases}0 & r \rightarrow \infty \\ F_{o} & r \rightarrow 0\end{cases}$

giving strings

$$
\begin{aligned}
& \langle\phi\rangle(r, \theta+\varphi)=h((\varphi+\theta) / 2) e^{-i F(r) T_{b}}\langle\phi\rangle_{o} e^{+i F(r) T_{b}} h^{-1}((\varphi+\theta) / 2) \quad \text { Dirac case } \\
& \langle\phi\rangle(r, \theta+\varphi)=h((\varphi+\theta) / 2) e^{-2 i F(r) \sigma_{z(12)}} h^{-1}((\varphi+\theta) / 2) \quad \text { Majorana case , }
\end{aligned}
$$

generalizing the untwisted strings of Equations (IV.1), (IV.2), and (IV.23). Note that this twist is distinct from the gauged monopole case: for the gauged monopole case, the plane of the string's internal space rotation rotates as we traverse the loop, locked to the loop angle $\theta$ to form a hedgehog configuration. Here, the textured string always rotates in the same internal space plane, assuming the same vacuum values; the entire configuration just shifts in $\varphi$ by an offset angle locked to $\theta$.

This offset of identical string configurations is essential in establishing nontrivial homotopy $\pi_{3}$. For each of our models varies nontrivially over, essentially, an $S U(2) \rightarrow S O(2)$ subspace of the model's symmetry-breaking pattern $G \rightarrow H$. For our $S O(6)$ model of section IIIA, our model varies over an $S O(4) \sim S U(2) \times S U(2)$ subspace, where $S U(2) \times S U(2) \rightarrow U(1) \times U(1)$; note that our model varies only over one $S U(2) \rightarrow U(1)$ factor, associated with the $J$-subalgebra. The canonical Alice model of section $\llbracket$ IIC is explicitly a symmetry breaking $S O(3) \rightarrow O(2)$. Finally, the BMS model of section varies over an $S U(2) \rightarrow S O(2)$ subspace of the symmetry-breaking $S U(N) \rightarrow S O(N)$.

In all these cases, then, we can exploit the isomorphism $S U(2) / S O(2) \sim S^{2}$ to identify the twisted loop's $\pi_{3}$ index with its Hopf number, the linking number of any two fibers of constant $\langle\phi\rangle$ in physical space. [10] Our twisted textured strings ( be exactly $2 \pi$-periodic in the angle $\varphi+\theta$ at finite $r$, and angle-independent at $r=0$. Thus we obtain one fiber by taking $r=0$ along the core of the loop, and a second by taking finite $r=r_{o}$, with $\theta+\varphi=0$. This second fiber, or constant value for $\langle\phi\rangle$, occurs at angle $\varphi=-\theta$ 
as we traverse the string loop taking $\theta$ from 0 to $2 \pi$. The two fibers are drawn below in Figure 2. Note that endpoints in $\theta$ and $\varphi$ are identified. The linking number is exactly one, showing that the twisted textured Alice string is a fundamental skyrmion. Thus, as in the gauged case, twisted textured Alice strings form fundamental point defects. Unfortunately, here the argument is not generic, as in the gauge case; it relies specifically on the Hopf map for the vacuum submanifold $S U(2) / U(1)$.

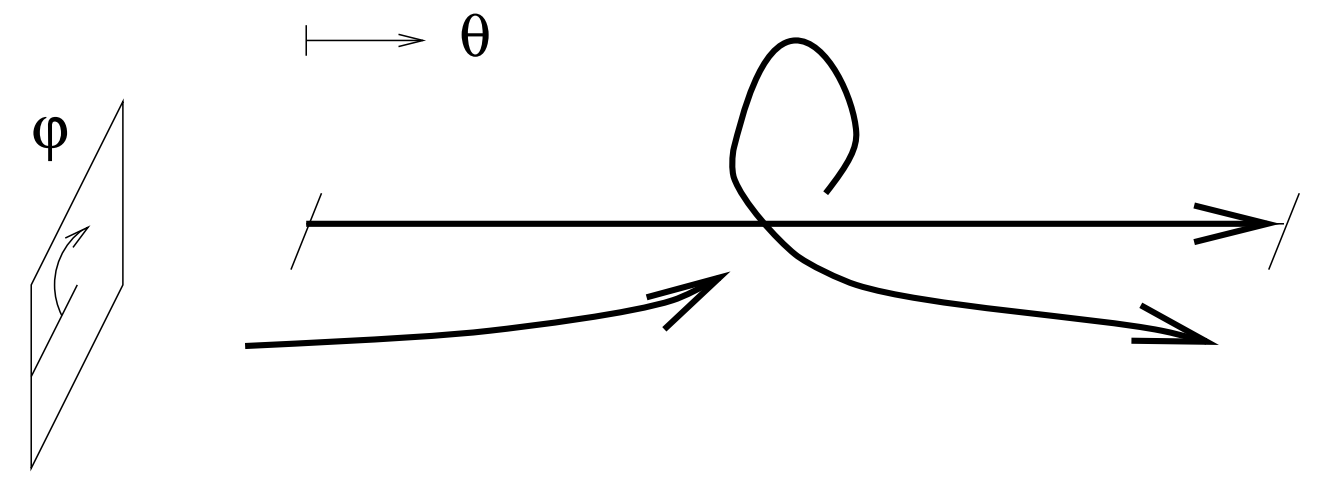

\section{Figure 2 : Linking of fibers for $\langle\phi\rangle$ : shown are $r=0$ and $r_{0}, \varphi=-\theta$ fibers. $r_{0}$ fiber crosses under then over $r=0$ fiber; ends are identified.}

\section{CONCLUSIONS}

We have demonstrated Alice behavior for global strings of both types: divergent global strings with nontrivial $\pi_{1}(G / H)$, and finite tension textured strings with nontrivial $\pi_{2}(G / H)$. In both cases, point defects emerge as twisted strings. Charge violation also emerges in both cases: in the first case, the global string inherits its Alice characteristics from its gauged counterpart; while in the second, charge violation is generic, at finite radius. Specifically, for the divergent global string, charge violation occurs for all generators that fail to commute with $W(2 \pi) U(2 \pi)$, where $U(2 \pi)$ is the Wilson loop of the associated gauged string, and $W(2 \pi)$ is a rare correction due to the nonabelian Aharonov Bohm effect. In the textured case, charge violation occurs for all generators which interact with the string at all, as a direct consequence of the nonabelian Aharonov Bohm effect. These textured strings are associated 
with topologically nontrivial loops in $H, h(\varphi)$. We showed that the nonabelian Aharonov Bohm effect determines an effective Wilson loop for the string at finite radius of $h(\alpha)$, where $\alpha$ ranges from 0 at infinite radius to $2 \pi$ at $r=0$. At finite radius, a mismatch between this Wilson loop, and locally defined unbroken symmetry generators, leads to generic and observable charge violation.

\section{ACKNOWLEDGMENTS}

This work was supported by NSF grant PHY-9631182, and by the University Research Committee of Emory University. I thank Tom Imbo for useful discussions, particularly on subtleties of the Hopf map. 


\section{REFERENCES}

[1] A. S. Schwarz, Nucl. Phys. B208 (1982) 141; A. Balachandran, F. Lizzi, V. Rodgers, Phys. Rev. Lett. 52 (1984) 1818.

[2] M. Alford, K. Benson, S. Coleman, J. March-Russell and F. Wilczek, Phys. Rev. Lett. 64 (1990) 1632; Nucl. Phys. B349 (1991) 414.

[3] J. Preskill and L. Krauss, Nucl. Phys. B341 (1990) 50; M. Bucher, H. K. Lo, and J. Preskill, Nucl. Phys. B386 (1992) 3.

[4] P. Nelson and S. Coleman, Nucl. Phys. B237 (1984) 1.

[5] K. Benson and T. Imbo, in preparation.

[6] K. Benson, A. Manohar, and M. Saadi, Phys. Rev. Lett. 74 (1995) 1932 (Available as hep-th/9409042); K. Benson and M. Saadi, Phys. Rev. D51 (1995) 3096 (Available as hep-th/9409109).

[7] P. McGraw, Phys. Rev. D50 (1994) 952.

[8] J. March-Russell and F. Wilczek, Phys. Rev. Lett. 68 (1992) 2567.

[9] F. Wilczek and A. Zee, Phys. Rev. Lett. 52 (1984) 2111.

[10] Y. Wu and A. Zee, Nucl. Phys. B324 623 (1989); F. Wilczek and A. Zee, Phys. Rev. Lett. 51, 2250 (1983). 